\title{
ANÁLISE DE DESDOBRAMENTO DE CUSTOS LOGÍSTICOS EM UMA GRANDE EMPRESA BRASILEIRA
}

\author{
ANALYSIS OF BREAKDOWN OF LOGISTIC COSTS IN A LARGE BRAZILIAN \\ COMPANY
}

\author{
Luis Fernando Terazzi - luis.terazzi@gmail.com \\ Marcelo Fachini Queiroz - marcelo_fachini@hotmail.com \\ João Batista de Camargo Junior - jbcjr@uol.com.br \\ Universidade de Araraquara (UNIARA) - Araraquara - São Paulo - Brasil
}

DOI: 10.31510/infa.v17i1.703

\begin{abstract}
RESUMO
$\mathrm{Na}$ busca por atender as necessidades dos consumidores de forma mais assertiva e, assim, conquistar vantagem competitiva, as empresas buscam desenvolver seus processos de gestão. Neste contexto, um modelo denominado de Manufatura de Classe Mundial pode contribuir para a melhoria das operações em uma organização. Um dos principais pilares desse modelo é o cost deployment, que auxilia na identificação de perdas em diversas áreas. Considerando a importância da logística para uma empresa, e também o fato dessa área ser um dos pilares do modelo, o objetivo desse trabalho é analisar como o cost deployment de logística é aplicado em uma grande empresa brasileira e quais principais perdas causais são identificadas por essa ferramenta. A metodologia utilizada para atingir esse propósito foi a pesquisa qualitativa, exploratória, por meio de um estudo de caso em uma multinacional de linha branca. Foram realizadas entrevistas com três gestores de três unidades produtivas da empresa, além de acompanhamento das operações. Os resultados do trabalho indicam que o cost deployment é aplicado a partir de cinco principais variáveis de custos logísticos, e que as principais perdas causais incluem embalagem inadequada, abastecimento de linha inadequado, distância do fornecedor, layout da planta e acuracidade de estoque.
\end{abstract}

Palavras-chave: Manufatura de classe mundial. Desdobramento de custos. Logística.

\begin{abstract}
In the quest to meet the needs of consumers in a more assertive way and thus gain competitive advantage, companies seek to develop their management processes. In this context, a model called World Class Manufacturing can contribute to the improvement of operations in an organization. One of the main pillars of this model is cost deployment, which helps to identify losses in several areas. Considering the importance of logistics for a company, and also the fact that this area is one of the pillars of the model, the objective of this work is to analyze how the cost deployment of logistics is applied in a large Brazilian company and which main causal losses are identified by this tool. The methodology used to achieve this purpose was qualitative, exploratory research, through a case study in a white line multinational. Interviews were conducted with three managers from three production units of the company, in addition to monitoring operations. The results of the work indicate that cost deployment is applied based on five main variables of logistical costs, and that the main causal losses include inadequate
\end{abstract}


packaging, inadequate line supply, distance from the supplier, plant layout and inventory accuracy.

Keywords: World-class manufacturing. Cost Deployment. Logistics.

\section{INTRODUÇÃO}

Considerando os requisitos dos consumidores, que estão cada vez mais rigorosos, e as características do mercado, muito mais concorrido e competitivo que em tempos anteriores, as organizações necessitam se tornar mais produtivas e flexíveis, melhorando continuamente seus produtos e serviços (FARIA; VIEIRA; PERETTI; 2012). Neste contexto, Flores, Faraco e Bond (2016) destacam que as organizações necessitam de mudanças nos sistemas de produção e de gestão para o seu desenvolvimento. Aliado ao desenvolvimento das empresas e visando um sistema mais eficiente de gestão de custos e gestão produtiva, surge o World Class Manufacturing (WCM) ou simplesmente Manufatura de Classe Mundial.

Segundo Palucha (2012), o WCM é um modelo de gestão integrado, utilizado pelos melhores sistemas produtivos do mundo, que se baseia no Sistema Toyota de Produção, elementos de Lean Manufacturing e Gestão da Qualidade Total. O WCM foi introduzido por Hayes e Wheelwright (1984), que descreveram o que foi desenvolvido pelas empresas japonesas e alemãs para competir nos mercados de exportação, e por Schonberger (1986), que cunhou o termo WCM a partir da experiência de empresas que tinham os melhores resultados nos aspectos de melhoria contínua e ágil.

A metodologia WCM é desenvolvida pelo aprofundamento de dez principais áreas da produção, chamadas de Pilares Técnicos, e de dez pilares de gestão, chamados de pilares gerenciais, que contém sete passos de implantação por pilar (FREITAS; FILHO, 2016). O segundo desses pilares técnicos é o Cost Deployment (CD) ou Desdobramento de Custos.

Considera-se que o Cost Deployment é um pilar fundamental e o que mais se distingue no modelo WCM porque ele se destaca nos aspectos de sistema de contabilidade de gestão e de sistema de medição de desempenho. É interessante perceber que o CD diferencia perdas de desperdícios. As perdas resultam do input que não é utilizado nos processos produtivos, enquanto que os desperdícios resultam no excesso de input na produção de output (CHIARINI; VAGNONI, 2015). Conforme Yamashina (2007), quanto mais detalhes as empresas possuírem para identificação das perdas, maior será a otimização de atividades específicas nos sistemas produtivos e em toda a cadeia de suprimentos. 
Já para Dudek (2013), um dos pilares técnicos mais importantes do WCM é o pilar de logística, que aborda os fluxos internos e externos através da integração da cadeia de suprimentos. É relevante notar que os pilares técnicos podem trabalhar em conjunto, e nesse sentido a aplicação do pilar de Cost Deployment no pilar de logística pode auxiliar as empresas a medir com eficácia seus custos logísticos e trabalhar na redução das perdas identificadas (YAMASHINA, 1999; EMBRACO, 2016; FIAT GROUP, 2011).

Paralelamente a esse raciocínio, Pessi (2017) aponta que o setor de Linha Branca passa por uma retração devido as incertezas atuais de mercado, e trabalha abaixo de sua capacidade produtiva desde 2013, quando o pico de produção foi atingido por conta da redução de impostos. Perante esse cenário, as maiores empresas atuantes nesse setor trabalham com ferramentas que visam o mais alto nível de desempenho nos processos, principalmente na logística que possui um fluxo complexo e oneroso. Nesse contexto, o objetivo deste trabalho é analisar como o Cost Deployment de logística é aplicado em uma organização de linha branca e quais principais perdas causais são identificadas por essa ferramenta.

Antes, entretanto, de realizar esta análise prática (cujos procedimentos metodológicos são descritos na seção 3 e os resultados na seção 4), a seção 2, que se inicia a seguir, apresenta uma fundamentação teórica que contempla o WCM e o CD inerentes a uma empresa, focando nos assuntos que sustentam o estudo que aqui se propõe.

\section{FUNDAMENTAÇÃO TEÓRICA}

\subsection{World Class Manufacturing (WCM)}

É possível afirmar que o WCM desenvolve as operações de uma organização especialmente através da aplicação de ferramentas e instrumentos, difundindo e padronizando os resultados alcançados (DUDEK, 2013). Assim para Yamashina (2007), a base do WCM é: a identificação do problema, a perda resultante deste problema, a escolha do método apropriado para reduzir a perda e o controle dos resultados. Portanto, para sustentar tal base, Palucha (2012) indica que os principais alvos do WCM devem ser a qualidade, custo, tempo de produção, flexibilidade e serviço ao cliente.

Em termos de estrutura, o WCM é dividido em pilares técnicos e pilares gerenciais,

conforme demonstra a Figura 1. Palucha (2012) afirma que os dez pilares técnicos 


\section{WNTERFACEETEENOLOGGCA}

correspondem ao detalhamento das áreas integradas de produção. Para Cortez et al. (2010), os pilares gerenciais indicam o comprometimento das pessoas e da organização na aplicação do modelo para auxiliar a alcançar os objetivos dos pilares técnicos.

Figura 1 - Pilares técnicos e gerenciais do WCM

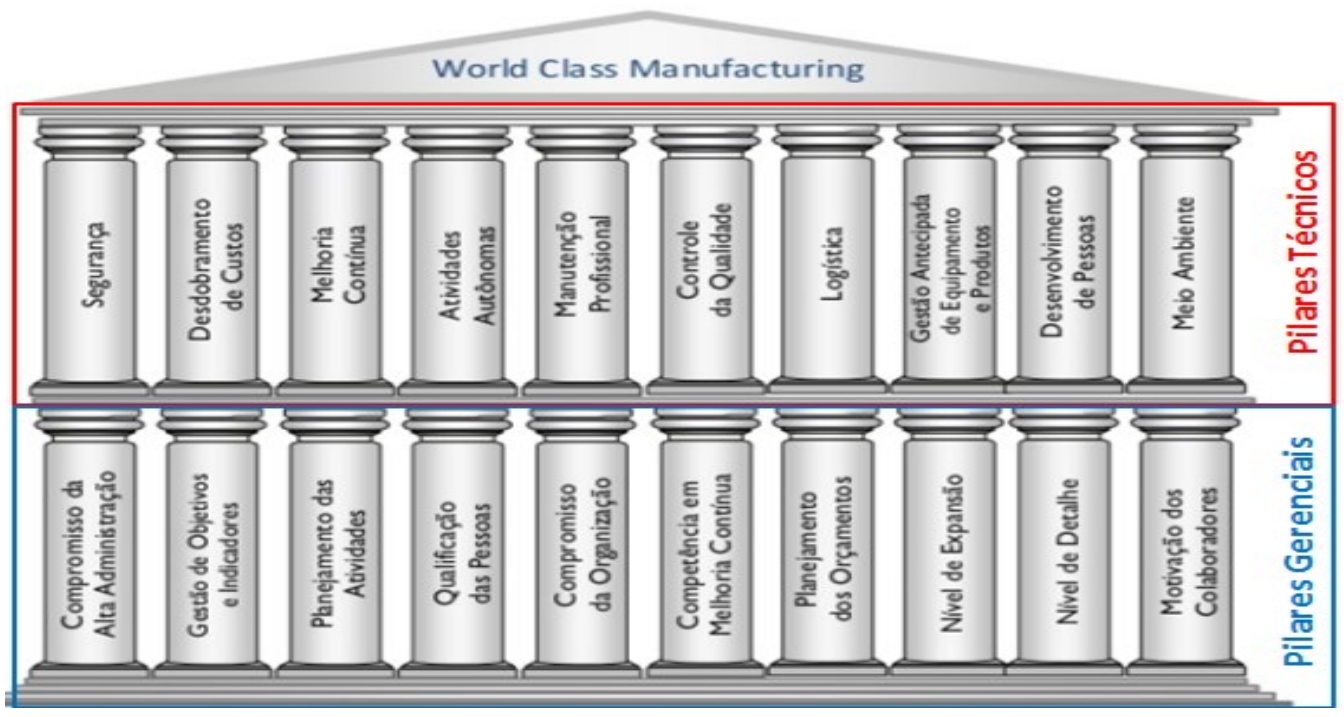

Fonte: adaptado de Yida (2014).

A avaliação das empresas em seus progressos no WCM é feita através de auditorias por especialistas externos, que indicam a certificação do nível alcançado. A pontuação que a organização pode receber varia de zero a cem, e para cada pilar técnico e gerencial é indicada uma nota em uma escala de 0 a 5 pontos. Para alcançar o nível bronze a empresa precisa receber no mínimo 50 pontos, enquanto para o nível prata o mínimo é de 60 pontos e para o nível ouro o mínimo é de 70 pontos. Para ser considerada nível World Class, a empresa precisa receber no mínimo 85 pontos (CORTEZ et al., 2010).

Torna-se importante ressaltar neste ponto que o foco deste trabalho se direciona sobre os pilares técnicos, especificamente em relação ao uso do CD na logística. Neste contexto, se destacam como resultados em um sistema de produção a redução dos níveis de estoques, minimização do deslocamento interno de materiais e diretrizes para integração de compras, produção e vendas (FELICE; PETRILLO, 2015). 


\subsection{Cost Deployment de Logística (CD)}

Segundo Yamashida (2007) o CD pode ser aplicado em diversas atividades de uma organização, como na melhoria contínua, controle de qualidade e desenvolvimento de pessoas, se transformando assim em uma ferramenta específica do WCM. Nesse sentido, o CD de logística é uma ferramenta utilizada para identificar e reduzir as perdas nos processos logísticos, sendo o direcionador do Pilar de Logística. Sua atuação envolve as cinco principais variáveis de custos logísticos nas empresas: mão de obra logística, equipamentos logísticos, área para estoque, frete e estoque de materiais (YAMASHINA, 2007).

$\mathrm{O} C D$ de logística consiste em sete etapas que são identificadas em matrizes. Essas matrizes possuem uma ordem de execução. Por exemplo, perdas e causas das perdas nos processos logísticos são identificadas em matrizes denominadas A, B e C. Os projetos para reduzir essas perdas são listados em uma matriz denominada $\mathrm{D}$, enquanto nas matrizes $\mathrm{E}, \mathrm{F}$ e G ocorrem a gestão desses projetos de redução de custos (GIOVANDO; CROVINI; VENTURINI, 2017). Ramos (2016) afirma que a matriz A é utilizada para localização das fontes de perdas logísticas e o impacto dessas perdas para cada processo. Essa matriz se baseia em dezoito perdas logísticas gerais, bem como em três perdas atribuídas ao transporte. Ela ajuda a identificar as principais perdas logísticas cruzando as vinte e uma perdas logísticas (linhas) com os processos logísticos (colunas), conforme demonstrado na Figura 2.

Figura 2 - Estrutura da Matriz A

\begin{tabular}{|c|c|c|c|c|c|}
\hline MACROCATEGORIA & CATEGORIA & PERDA & Processo 1 & Processo 2 & Processo 3 \\
\hline \multirow{3}{*}{$\begin{array}{l}\text { 岁 } \\
\text { 음 } \\
\text { 严 }\end{array}$} & Perda por Estoque & $\begin{array}{l}\text { Perda de estoque por excesso } \\
\text { Perda de estoque por segurança } \\
\text { Perda de estoque minimo }\end{array}$ & & & \\
\hline & $\begin{array}{l}\text { Perda por mão de } \\
\text { obra }\end{array}$ & $\begin{array}{l}\text { Perda mão de obra por excesso } \\
\text { Perda mão de obra eficiência } \\
\text { Perda mão de obra minima }\end{array}$ & & & \\
\hline & Perda por espaço & $\begin{array}{l}\text { Perda espaço por excesso } \\
\text { Perda espaço por eficiência } \\
\text { Perda espaço minimo necessário }\end{array}$ & & & \\
\hline \multirow{3}{*}{ 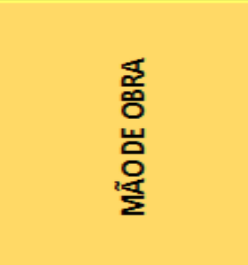 } & $\begin{array}{l}\text { Perda por mão de } \\
\text { obra }\end{array}$ & $\begin{array}{l}\text { Perda mão de obra por excesso } \\
\text { Perda mão de obra eficiência } \\
\text { Perda mão de obra minima }\end{array}$ & & & \\
\hline & Perda por espaço & $\begin{array}{l}\text { Perda espaço por excesso } \\
\text { Perda espaço por eficiência } \\
\text { Perda espaço minimo necessário }\end{array}$ & & & \\
\hline & $\begin{array}{l}\text { Perda por } \\
\text { equipamento }\end{array}$ & $\begin{array}{l}\text { Perda equipamento por excesso } \\
\text { Perda equipamento eficiência } \\
\text { Perda equipamento minimo }\end{array}$ & & & \\
\hline TRANSPORTE & $\begin{array}{l}\text { Perda por } \\
\text { transporte }\end{array}$ & $\begin{array}{l}\text { Perda transporte por excesso } \\
\text { Perda transporte por eficiência } \\
\text { Perda transporte minimo }\end{array}$ & & & \\
\hline
\end{tabular}

Fonte: adaptado de Fiat Group (2011). 
A matriz B é importante para distinguir perdas causais e resultantes. Ela atribui perdas resultantes a perdas causais, conforme suas inter-relações, e deve ser confeccionada por um time multifuncional que inclui desde o gerente até operadores experientes. A quantificação das relações de causa e efeito entre perdas deve ser uma distribuição fracionária das perdas resultantes para perdas causais (HOEG; KNUTSEN, 2016). Segundo Yamashina (2007), para construir a matriz B é necessário identificar todas as possíveis perdas causais que estão gerando as perdas. A matriz B atravessará então as causas (linhas) com as perdas logísticas mais relevantes (definindo um valor limiar) apontadas na matriz A (colunas) conforme demonstrado na Figura 3.

Figura 3 - Matriz B: Perdas casuais e perdas resultantes

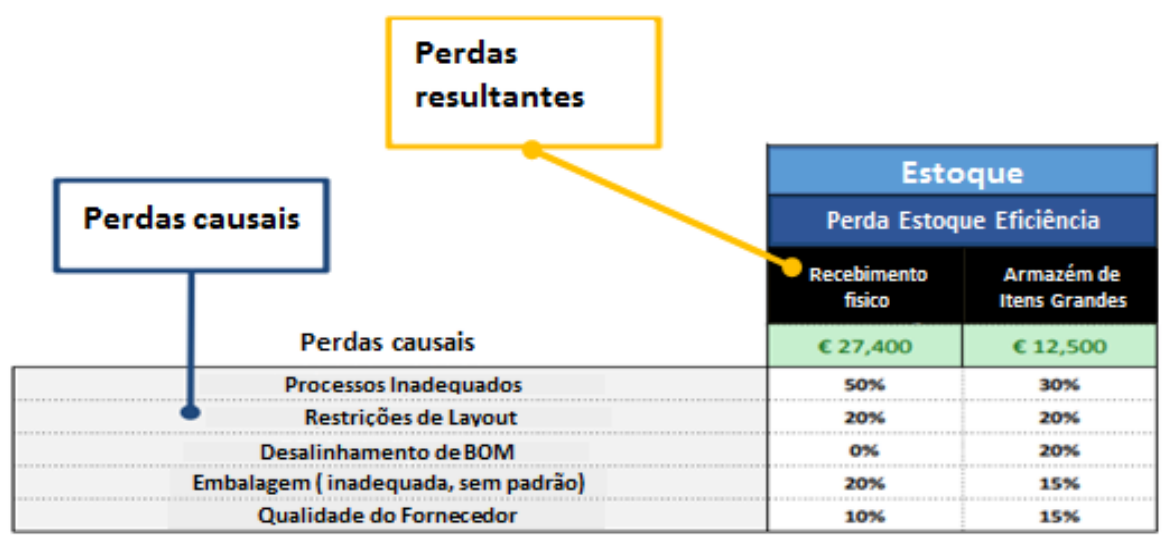

Fonte: adaptado de Fiat Group (2011).

A matriz C, por sua vez, expressa todas as perdas em termos monetários a fim de permitir a visualização dos custos reais incorridos nessas perdas. Ela apresenta o custo total atribuído a cada perda causal, e deve ser confeccionada pelo time técnico da logística sob supervisão do setor de Controladoria (HOEG; KNUTSEN, 2016). Segundo Yamashina (2007), o propósito da matriz $\mathrm{C}$ é destacar as perdas causais e processos, considerando que os maiores montantes de perdas foram identificados nas matrizes A e B. A preparação da Matriz C consiste em reorganizar os dados da Matriz B, agrupando as perdas resultantes por causas raiz e processo e, em seguida, dividir os valores por tipo de custo, conforme Figura 4. 


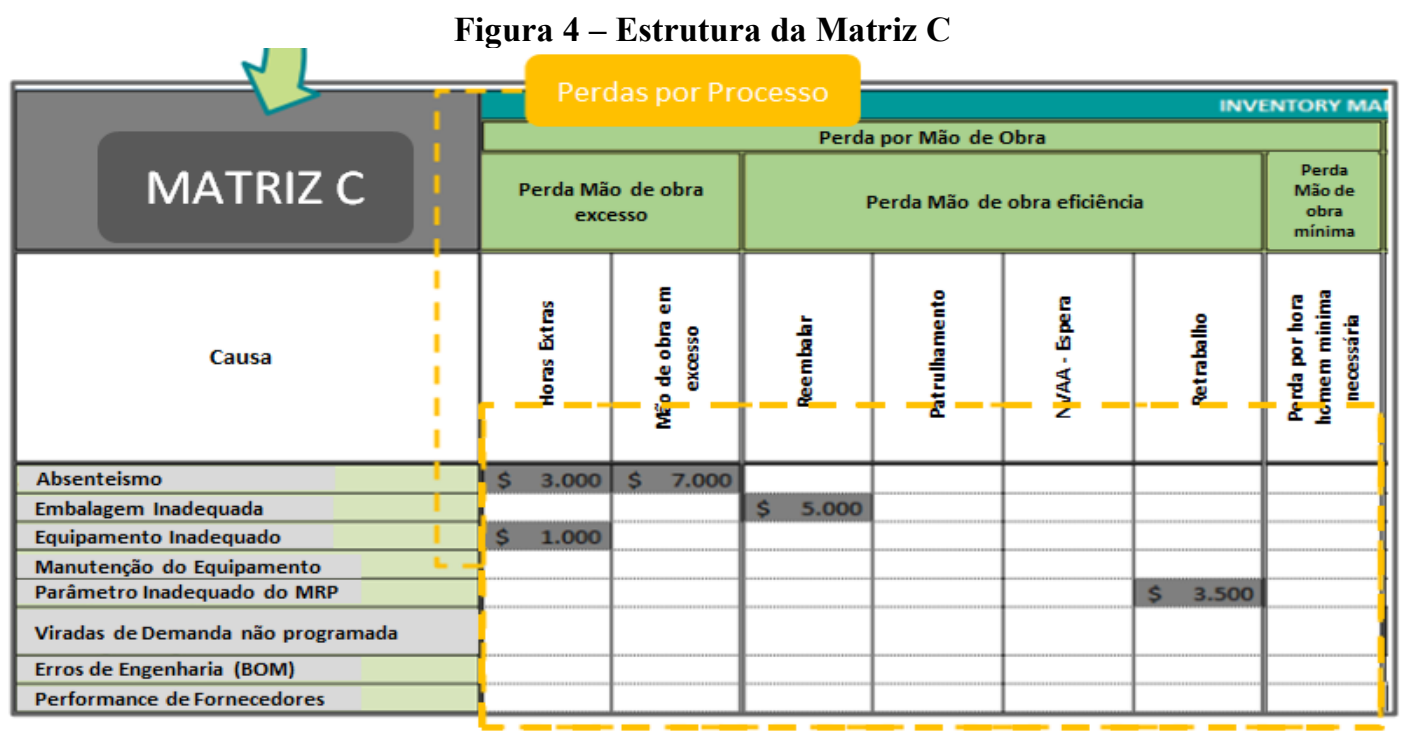

Fonte: adaptado de Embraco (2016).

$\mathrm{Na}$ matriz D é estabelecida a prioridade de ações corretivas e as ferramentas para evitar perdas por meio do método Impacto, Custo e a Facilidade (ICF), que envolve avaliar a ocorrência das perdas causais mais importantes identificadas na Matriz C. Já na matriz E é realizado um balanço econômico entre custo de implementação do novo método e o benefício derivado da redução das perdas, sendo possível optar por qual melhoria iniciar. As matrizes $\mathrm{F}$ e $\mathrm{G}$ gerenciam as melhorias visando eliminação ou redução das perdas em cada área, mensurando também a redução financeira (GIOVANDO; CROVINI; VENTURINI, 2017).

Desse modo, é possível observar que o CD de Logística torna os processos de tomada de decisão mais eficazes e profissionais. Uma limitação à implementação dessa ferramenta é referente aos recursos substanciais para uma coleta de dados rigorosa. Sua aplicação bemsucedida requer a entrada de dados confiáveis e, sendo assim, as organizações necessitam desenvolver rotinas para melhorar e modificar continuamente sua coleta de dados (NETLAND; HOEG; KNUTSEN, 2016).

\section{PROCEDIMENTOS METODOLÓGICOS}

O presente trabalho é classificado como estudo de caso de natureza qualitativa e de caráter exploratório. Para Yin (2010), o estudo de caso é uma ferramenta de pesquisa que auxilia na investigação de fenômenos dentro de um contexto real e o estudo qualitativo caracteriza-se pela análise em profundidade do objeto de interesse do pesquisador sem o uso de instrumental estatístico para a análise dos dados. Segundo Gil (2010), a pesquisa exploratória tem a 
finalidade de proporcionar informações sobre o assunto estudado, possibilitando sua definição e delineamento. A investigação foi desenvolvida, inicialmente, através de pesquisa bibliográfica, que segundo Gil (2010) se refere à busca e utilização de referências teóricas publicadas com o objetivo de recolher informações ou conhecimentos prévios sobre o problema.

O objeto de estudo é uma empresa do setor de linha branca que se posiciona entre as principais fabricantes de eletrodomésticos. Ela foi escolhida por ser uma organização multinacional, por possuir com um fluxo logístico intenso e principalmente por utilizar o WCM desde 2017. A organização possui cerca de 11.000 funcionários distribuídos em três unidades produtivas, localizadas no estado de São Paulo, Santa Catarina e Manaus. A unidade produtiva de São Paulo produz lavadoras e fogões, a de Santa Catarina produz refrigeradores e a unidade de Manaus dedica-se a produção de forno micro-ondas.

Para a coleta de dados foi elaborado um roteiro de entrevista semiestruturado baseado na pesquisa bibliográfica conduzida. As entrevistas foram realizadas com os responsáveis pela aplicação do CD de logística, identificados no Quadro 1, nas três unidades produtivas da empresa e tiveram duração média de quarenta minutos cada. Vale ressaltar que as entrevistas foram realizadas através de vídeo chamada durante o mês de dezembro de 2019.

Quadro 1 - Relação de colaboradores entrevistados

\begin{tabular}{|c|c|c|}
\hline Estado da unidade & Tempo de empresa & Grau de Instrução \\
\hline Santa Catarina/SC & 15 anos & Engenheiro de Produção \\
\hline Amazonas/AM & 5 anos & Engenheiro de Produção \\
\hline São Paulo/SP & 10 anos & Engenheiro de Logística \\
\hline
\end{tabular}

Fonte: autoria própria.

Os entrevistados foram selecionados por pertencer ao setor de engenharia logística da empresa e, por isso, terem contato direto com o CD de logística. É importante ressaltar que na fase de coleta de dados o foco do trabalho estava nas perdas causais da empresa, e não nas perdas resultantes delas derivadas. Isso se deu em função desse último tipo de perda se referir a valores monetários, que fogem da proposta da pesquisa. Na etapa final de análise de dados, buscou-se correlacionar a literatura apresentada no referencial teórico com os resultados obtidos a fim de atingir o objetivo proposto. 


\section{RESULTADOS E DISCUSSÃO}

Durante as entrevistas foi constatado que a inserção dos valores nas matrizes das três plantas ainda é manual para as cinco variáveis logísticas do $\mathrm{CD}$. Na definição dos processos a serem adicionados na matriz $\mathrm{A}$ as três plantas determinaram atividades que vão do recebimento dos materiais até o abastecimento nas linhas de montagem. Os processos levantados nessa matriz são diferentes entre as plantas e variam de acordo com o tamanho e características de cada uma, seguindo as sete etapas das matrizes.

As maiores perdas identificadas nas plantas do estado de São Paulo e de Santa Catarina estão contidas nas variáveis frete e mão de obra. Para a planta de Manaus, a variável espaço possui valores elevados por se tratar de uma fábrica com grande estoque de material importado em kits que demandam uma área excedente para estoque.

A identificação das perdas causais e a relação entre causas e efeitos das perdas na matriz B são realizadas manualmente por um time multifuncional. Os efeitos das perdas causais (as perdas resultantes, em valores monetários) são distribuídos nos diferentes processos listados nas unidades da empresa estudada. Conforme demonstrado por Yamashina (2007), para construir a matriz B é necessário identificar todas as possíveis perdas causais e então cruzá-las com as perdas dos processos identificados na matriz A, gerando as perdas resultantes. Identificou-se na entrevista relaciona ativamente às 21 perdas logísticas da matriz $\mathrm{A}$. Como exemplo, na unidade do estado de São Paulo a matriz A conta com 10 processos, que se cruzam com as 21 perdas logísticas resultando em 210 perdas resultantes.

Nota-se que as perdas causais relacionadas na Matriz B foram iguais para as três plantas estudadas, conforme demonstra o Quadro 1. Segundo os entrevistados, esse fato pode interferir nos resultados da aplicação do CD de logística na empresa, uma vez que cada planta pode contar com perdas causais específicas que não foram corretamente identificadas. De acordo com Hoeg e Knutsen (2016), a identificação de todas as possíveis perdas causais e de suas respectivas perdas resultantes é muito importante para a eficácia da implantação do CD.

Quadro 1 - Lista de perdas causais das plantas estudadas

\begin{tabular}{|c|c|c|}
\hline Abastecimento de Linha Inadequado & Fraqueza de Processo & Distância do Fornecedor \\
\hline Embalagem Inadequada & Layout da Planta & Acuracidade de Estoque \\
\hline Equipamento Inadequado & Peças com defeito e Scrap & Estratégia de Compras \\
\hline
\end{tabular}




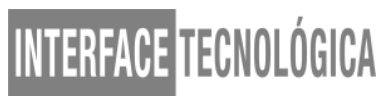

\begin{tabular}{|c|c|c|}
\hline Parâmetros de MRP Inadequados & Excesso de Produção & Sistema de Chamada de Materiais \\
\hline Trocas na Programação de Produção & Atraso no Trânsito & Excesso de Manuseio de Material \\
\hline Erros de Engenharia & Nivelamento de Produção & Infraestrutura e Tecnologias de \\
& & TI \\
\hline Perda por Estoque & Performance dos Fornecedores & Excesso de Estoque \\
\hline Falta de 5S e 5T & Falta de Treinamento e Atenção & Absenteísmo \\
\hline
\end{tabular}

Fonte: Elaborado pelos autores.

A perda com maior valor monetário na Matriz C, apontada pelos entrevistados, nas plantas estudadas é a de embalagem inadequada. Isso porque essa causa raiz, impacta nas cinco variáveis do $\mathrm{CD}$, e foi a principal fonte dos projetos de redução de custo. No entanto, em relação aos processos de fabricação, os entrevistados destacaram a logística externa com o maior custo, destacando o frete e o estoque em trânsito. Já os custos dos processos relacionados a estoque e equipamentos foram moderados nas três unidades produtivas. Portanto, conclui-se que o objetivo da matriz C proposto por Yamashina (1999) é atingido nas três unidades, uma vez que as três plantas conseguem quantificar, mensurar e transformar em valor as perdas identificadas nas matrizes A e B.

A matriz D é utilizada pelas três plantas para a definição de projetos de melhoria, entretanto as matrizes E, F e G ainda não foram confeccionadas e a gestão dos projetos é realizada em planilhas avulsas em todas as plantas pelo time de controladoria. Foi citado pelos entrevistados que essas planilhas alimentam as matrizes E, F e G do CD de logística. Em geral, as matrizes são atualizadas semestralmente, e a cada revisão os entrevistados reportaram que o nível de detalhe e a maturidade dos dados são melhorados. Ressalta-se que as plantas estão no início da implementação da ferramenta e, por isso, atualizaram apenas duas vezes as matrizes.

Em relação como o Cost Deployment de logística é aplicado, o maior obstáculo reportado pelos profissionais é o preenchimento da matriz $B$, já que a relação entre causas e efeitos (perdas causais e resultantes) ainda é realizada manualmente e demanda muito tempo e trabalho. Esse preenchimento manual gera, além das dificuldades citadas, dados menos confiáveis para as análises de custo. Assim, constata-se que a afirmação realizada por Netland, Hoeg e Knutsen (2016) de que aplicação da ferramenta requer a entrada de dados confiáveis é verdadeira, e fortalece a premissa de que as empresas precisam de rotinas que melhorem continuamente suas análises de dados para efetividade do CD de logística.

Neste contexto, todos os entrevistados relataram que as matrizes ajudam a empresa 
estudada a entender onde estão seus maiores custos e perdas nos processos logísticos, e conseguem direcionar os recursos e esforços de forma mais assertiva e eficiente. Com as matrizes, os projetos de redução de custos trouxeram impactos favoráveis para a organização. Assim, os profissionais entrevistados acreditam que com o passar do tempo o CD será ainda mais eficaz e determinante para manter a competitividade da empresa.

\section{CONSIDERAÇÕES FINAIS}

O objetivo desta pesquisa foi de analisar como o CD de Logística é aplicado em uma organização de linha branca e quais as principais perdas causais que são identificadas por essa ferramenta. Por meio da análise do estudo de caso, pode-se concluir que o CD foi utilizado na empresa analisada através da inserção manual de dados das cinco principais variáveis de custos logísticos. Os processos foram definidos conforme a complexidade de cada planta, e a identificação das perdas causais e sua relação com os processos foram realizadas também de forma manual através de um time multifuncional de cada unidade produtiva da empresa estudada. Outro fato relevante identificado sobre a aplicação do CD na empresa é que as três plantas identificaram o mesmo conjunto de 24 perdas causais, o que segundo os entrevistados demonstra a possibilidade de falha na identificação individual de perdas e possível interferência nos resultados do CD.

Em relação a descoberta das perdas casuais, a principal identificada pelo CD se relaciona com a embalagem inadequada nas três unidades produtivas. Outras perdas identificadas incluem distância do fornecedor, layout da planta, acuracidade de estoque, equipamento inadequado, peças com defeito, falta de treinamento, entre outras.

Tratando-se de um estudo de caso único, a principal limitação deste trabalho é não poder ser generalizado. Quanto a pesquisa em si, não se considera que houve limitações uma vez que os pesquisadores tiveram total acesso aos entrevistados. Como pesquisas futuras, é possível dar continuidade a esse trabalho considerando outros segmentos industriais. Outra proposta é avaliar o uso do CD de logística em empresas que estejam em diferentes níveis de implementação do WCM no intuito de visualizar diferenças e possíveis melhorias que possam ser implementadas. 


\section{REFERÊNCIAS}

CHIARINI, A.; VAGNONI, E. World-class manufacturing by Fiat: Comparison with Toyota production system from a strategic management, management accounting, operations management and performance measurement dimension. International Journal of Production Research, v. 53, n. 2, p. 590-606, 2015.

CORTEZ, P. R. L.; BACHOUR, M. C.; PEREIRA, M. C.; DIAS, A. V. C.; BAGNO, R. B. Análise das relações entre o processo de inovação na engenharia de produto e as ferramentas do WCM: estudo de caso em uma empresa do setor automobilístico. XXX ENEGEP. São Carlos, 2010.

DUDEK, M. Organizing of Logistic Pillar in WCM Systems. Carpathian Logistics Congress: Poland, 2013.

EMBRACO. Logistics Cost Deployment. WCM Central Team: Joinville, 2016.

FARIA, A.C.; VIEIRA, V.S.; PERETTI, L.C.; Redução de custos sob a ótica da manufatura enxuta em empresa de autopeças. Revista Gestão Industrial. v. 08, n. 02: p. 186-208, 2012.

FELICE, F.; PETRILLO, A. Optimization of Manufacturing System through World Class Manufacturing. IFAC-Papers Online, 48(3), pp.741-746, 2015.

FIAT GROUP. Logistics Cost Deployment. Supply Chain Management: Industrial Logistics Central Team, Betim, Vol.3, 2011.

FLORES, R.E.; FARACO, N.N.T; BOND, D. Proposta de Metodologia para Construção da ferramenta de análise de falhas no contexto do WCM (World Class Manufacturing).

Encontro Nacional de Engenharia de Produção. v.36, n.1, p.1-12, 2016.

FREITAS, I.S.; FILHO, L. C. B. Diagnóstico da implantação da Metodologia de Gestão Estratégica World Class Manufacturing nas indústrias de Pernambuco. Revista de Engenharia e Pesquisa Aplicada, v.3, n.1, 2016.

GIOVANDO, G.; CROVINI, C; VENTURINI, S. Cost Deployment Implementation: a case study. 10th Annual Conference of the EuroMed Academy of Business, 2017.

HAYES, R.H.; WHEELWRIGHT, S.C. Restoring our Competitive Edge Competing Through Manufacturing, Jhon Wiley \& Sons, Inc., USA, 1984.

HOEG P. C. H.; KNUTSEN D. H. Roadmap for Manufacturing Cost Deployment. NTNU, Trondheim. Copycat, 2016.

NETLAND, T.H.; HOEG, P.C.H.; KNUTSEN, D.H. Manufacturing Cost Deployment: How to select the right projects, 2016. 
PALUCHA, K. World Class Manufacturing Model in Production Management. International Scientific Journal, Poland, v. 58, n. 2, p.227-234, Dec. 2012.

PESSI, C.E.A.S. Gestão da cadeia de suprimentos: um estudo de caso em uma multinacional de linha branca. $54 \mathrm{f}$. Monografia de Conclusão de Curso. Engenharia de Transportes e Logística, Universidade Federal de Santa Catarina, Joinville. Santa Catarina. Brasil, 2017.

RAMOS, P.S. World Class Manufacturing: Pilar Cost Deployment. Como e o que implementar. Universidade de Aveiro. Departamento de gestão, economia, engenharia industrial e turismo, 2016

SCHONBERGER, R.J. World class manufacturing: the lessons of simplicity applied, New York: Free Press, 205 p., 1986.

YAMASHINA, H. Challenge to world class manufacturing. International Journal of Quality of Reliability Management, v. 12(34), 30-31, 2007.

YAMASHINA H. Manufacturing Cost Deployment. Journal of the Japan Society for Precision Engineering, v. 65, n. 2, p.260-266, 1999.

YIDA, P. Manufatura de Classe Mundial. In: SIMPÓSIO SAE BRASIL DE MANUFATURA DE CLASSE MUNDIAL, v. 9, Piracicaba, 2014.

YIN, R. K. Estudo de casos: planejamento e métodos. Bookman: Porto Alegre - RS, 2010. 